\title{
ON THE IMPEDANCE DUE TO SYNCHROTRON RADIATION
}

\author{
S. HEIFETS \\ Stanford Linear Accelentor Center, Stanford University. Stanford, CA 94909 USA \\ and
}

A. MICHAILICHENKO

Institute of Nuclear Physics, Novosibarsk, USSR

Abstract

A qualitative consideration of the impedance caused by the synchrotron radiation is given. The rigorous results such as the value of the threshold frequency and the maximum value of the impedance are obtained in a simple way.

\section{INTRODUCTION}

The problem of the synchrotron radiation of a charge in a conductive vacuum chamber has been considered many times. see references in recent publications $[1,2,2]$. The rigorous consideration is based on the exact solution of the wave equation in a particular geometry (a charge moving between two conductive plenes or in a toroidal chamber) and involves rather cumbersome calculations. Providing very useful reference models, these solutions call for a more simple heuristic picture of the physics involved which would clarify the situation, especially in cases where the exact solution is unknown.

Consider, for example, the results [2] for a charge moving along a circle with the radius $R$, in a pillbox cavity with the radius $b$ and the height $h=2 g$. The real part of the impedance is given as a sum of d-functions due to the excitation of eigen modes of the cavity. The threshold frequency $w_{3} h$ is much higher than the cutoff frequency isu: $=\pi c / h$,

$$
\omega_{t h} \simeq \frac{c}{R}\left(\pi \frac{R}{h}\right)^{3 / 2} \gg 1 .
$$

and the maximum value of the impedance

$$
\left[\operatorname{Re} \frac{Z(n)}{n}\right]_{\max } \simeq 300 \frac{g}{R} \mathrm{Ohn}
$$

is independent of $b$, see Appendix. This indicates that consideration based on the modal analysis is superfluous while the threshoid frequency is a result of the intrinsie properties of the synchrotron radiation in a wareguide.

The threshold frequency: may be obtained in the following way. As it is well known, the harmonic $n$ of the synchrotron radiation can be radiaced only within the small angle $\theta$ with the plane of motion:

$$
\theta<\pi^{-1 / 3} \text {. }
$$

That follows from the intensity of the n-th harmonic of the synchrotron radiation [4] of an ulerarelativistic particle $\gamma>1$ :

$$
\begin{aligned}
& d W(n, \theta)=\frac{c e^{2}}{R} \frac{n^{2}}{6 \pi^{3}} \\
& \quad \times\left\{\epsilon^{2} K_{2 / 3}^{2}\left(\frac{n}{3} \epsilon^{3 / 2}\right)+\epsilon \cos ^{2} \theta K_{1 / 3}^{2}\left(\frac{n}{3} e^{3 / 2}\right)\right\}
\end{aligned}
$$

where $\epsilon^{2}=1 / \gamma^{2}+\theta^{2}$. The intensity rolls off exponentially for harmonics

Work supported by Department of Energy contract DE-

$$
n>\frac{3}{2}\left(\frac{1}{\gamma^{2}}+\theta^{2}\right)^{-3 / 2}
$$

in agreement with Eq. (1). The result Eq. (1) is the direst result of the Lorentz transformation of the dipole radiation in the moving frame of a particle and is an intrinsic feature of the synchrotron radiation.

Consider now a particle moving in $(x, y)$ plane between two conductive planes separated by the distance $h=2 \mathrm{y}$. The radiated wave propag ites between the planes as in a waveguide. Usually, this $i$ sossible if the ware frequency $\omega$ is above the cutoff frequ $c y: k=\omega / c>\pi / h$. For the waves with irequencies well cbove the cutoff frequency the propagation of the wave may be described in terms of the geometric optics with a wave: $\operatorname{ctor} \vec{k},|\vec{k}|=\kappa / c=n 3 / R$. The boundary conditions on the conductive walls still require that the vestical component of the wave sector cannot be too small:

$$
k_{\perp}=k \theta>\frac{\pi}{h} \text {. }
$$

The cut of frequency corresponds to the angle $\theta \simeq 1$. For the harmonics $n>>1$ the angle $;$ restricted by $\mathrm{Eq}$. (1). Eqs. (1) and (4) give the threshol: frequency:

$$
n_{t h}=\frac{\omega R}{c}=\sqrt{\frac{2}{3}}\left(-\frac{R}{h}\right)^{3 / 2}
$$

The synchrotron radiation with $n<n_{t h}$ may be radiated only with the radjation angle $\theta>n^{-1 / 3}$, otherwise the boundary conditions cannol be satisfied. The probability of such radiation, as has been mentioned above, is expenentially small.

Hence, the single particle synchrotron radiation is exponentially small (see Eq. (2)) for harmonics $n<n_{t h}$ and. as usually, with $n>n_{\text {mos }}=\boldsymbol{\gamma}^{3}$. Unfortunately, it is always $n_{t A}<<n_{m a x}$, and the decrease of the total radiativ! power due to suppression of the radiation witla the harmonics $n<n_{t h}$ is small.

The parameters defining the threshold frequency have to be clarified for more complicated structures such an a Loroidal chamber, where there are several geomet ric dimel.sions (the height and the width of the chamber). Nith is good accuracy the polarization of the synchrotron radjittion is such that the vector of the electric field is in the plane of motion (intensity of this polarization is $i / 8$ of the total intensity [4]). Therefore, only the height of tle' chamber enters in the boundary conditions for the tangential component of the electric field and in the thresliold frequency.

It is worthwhile to consider the radialion length of $I_{1}$. mode $n$. As usual, the radiation length or the length of th. formation of the radiation can be defined as the lengl $\mathbf{l}$.

AC03-76SF00515. 
where the phase of the radiation remains small: $\mid k_{i} L-$ $-1 t \mid<\pi$. Using $k_{j}=(n / R) \cos \theta$, and $\mathrm{Eq}_{q}$. (3) we obtain:

$$
L=2 \pi R n^{-1 / 3}=2 \sqrt{\pi h R} .
$$

The length $\mathrm{L}$ is small, $L \ll R$. Hence, the results obtained for a periodic motion on a circle with the radius $R$ are valid also for an aperiodic motion or a periodic motion along a complicated trajectory with $\mathrm{R}$ being a local radius provided $R>L \simeq \sqrt{h R}$.

It should be noted that the parameter $\omega_{0}=\mathrm{c} / R$ has a meaning of a fundamental frequency of oscillations. Thus. the same effect of suppression of the radiation below a threshold frequency can be exfected for a dipole oscillating with frequency $\omega_{0}$ and propagating in a straight waveguide.

The effect of the finite conductivity can be estimated comparing the radiation length $\mathrm{Eq}$. (6) with the absorption length of a wave. The absorption length $L_{a j}$ is defined as the length where the intensity of a wave decreases by a factor of $e$. It can be estimated from the definition of a $Q$-factor of a mode, $L_{a b s}=c t=c /(Q+)$ with $Q=a / \delta$. Here $\delta$ is the skin depth, and $a$ is the geometric factor of the order of the beam pipe aperture. Clearly, the effect of finite conductivity is small provided $L_{a b}>L$.

Let us consider now the coherent radiation of a bunch. The radiation of a bunch can be coherent if the bunch length is small compared with the wavelength. For a Gaussian bunch with ims length $\sigma$ that means $\omega \sigma / c<1$ or $n<R / \sigma$. Because radiation of the modes $n<n_{t h}$ is suppressed, the coherent synchrotron radiation is possible only for very short bunches:

$$
\sigma<\frac{h}{\pi} \sqrt{\frac{3}{2}} \sqrt{\frac{h}{\pi R}} .
$$

\section{I. Impedance DUe to SYNChrotron Radiation}

The electromagnetic field of the synchrotron radiation of a particle may change the energy of other particles in a bunch. The energy variation is described usually in terms of the wake field or, in the case of two point-like particles separated by the distance $s$, in terms of the $\delta$ - functional walie field $W(s)$. The last is related to the variation of the energy of the second particle $\Delta E$ due to interaction with the tangential to the trajectory component of the field of the first particle. For a periodic motion in a plane $z=0$ on a circle with the radius $R, \Delta E(s)$ is the variation of the energy per turn due to the arinuthal harmonic of the electric field:

$$
\begin{aligned}
W(s) & =-\left(\frac{1}{e^{2}}\right) \Delta E(s) \\
& =-\frac{c}{e} \int_{0}^{T} \operatorname{dt} E_{\phi}\left(R, \phi=\frac{\omega_{0} t-s}{R, z=0, l}\right) .
\end{aligned}
$$

Here $T=2 \pi R / v=2 \pi / \omega_{0}$ is the revolution period, and $\omega_{0} / 2 \pi$ is the revolution frequency. The field $E_{\theta}(r, \phi, z, t)$ is periodic in time and azimuth:

$$
E_{\phi}(r, z, \phi, t)=\int \frac{d \omega}{2 \pi} e^{-i \omega t} \sum_{n} e^{i n \phi} E_{n}(r, z, \omega)
$$

wilh harmonics

$$
E_{n}(r, z, \omega)=\omega_{0} \sum_{\nu} E_{n, \nu} \delta(\omega-n \nu) .
$$

Eqs. (8) and (10) define the wake in terms of the lartuminte $E_{n, \nu}(\tau, z)$ :

$$
W(s)=-\frac{c}{e} E_{n, n}(R, 0) e^{-\ln s / R}
$$

The longitudinal beam impedance for a periodi: 111,1611

$$
Z(\omega)=Z_{n} \omega_{0} \delta\left(\omega-\pi \omega_{0}\right)
$$

is the Fourier harmonic of the periodic $W(s)=\| v(s+2-k$. wake:

$$
W(s)=\sum_{n} \frac{Z_{n} \omega_{0}}{2 \pi} e^{-i n \omega_{i} s / n}
$$

Hence

$$
Z_{n}=\frac{2 \pi R}{e} E_{n, n}(R, 0)
$$

Note that $Z_{n}$ is the average value of the impedance. Eq. (12):

$$
\left\langle Z\left(\omega^{\circ}\right)\right\rangle=\frac{1}{\omega_{0}} \int_{\omega_{0}} d \cdot Z\left(\omega^{*}\right)=Z_{n}
$$

where $n=\omega / \omega_{0}$ and the interval of the averaging is -

The azimutbal component of the electric field of at point-like charge e moving with velocity $v$ on a circle with the radius $R$ in the $(x, y)$ plane has harmonies

$$
\begin{aligned}
& E_{n, \nu}^{\infty}(r, z)=\frac{e \omega_{0}}{2 \pi} \int d \phi e^{-i n \phi} \int_{0}^{T} d t e^{i r_{\infty} d} \\
& x\left\{\frac{i k v}{c} \cos \left(v_{0} t-\phi\right)-\frac{1}{r} \frac{\partial}{\partial 0}\right\} \frac{r^{2 k n}}{r}
\end{aligned}
$$

where $\rho=|\vec{r}-\vec{r}(t)| .|\vec{r}(t)|=R$, and the integration ove 0 is performed on tlie interval $2 \pi$.

The harmonic $E_{n, n}(R, 0)$ defines the impedance. Substituting $\rho=2 R \sin (\alpha)$. where $\alpha=2\left(0-\omega_{0} t\right)$ we obtain.

$\operatorname{Re} \frac{Z(n)}{n}=\frac{Z_{0}}{2} \int_{0}^{\pi} d a\left[3^{2} \cos 2 a-1\right\} \frac{\sin \{2 n 3 \sin a-2 n a\}}{\sin n}$

Here $\beta=v / c$, and $Z_{0}=120 \pi$ Ohm. The modes of interest are the modes $1<<n<<\gamma^{3}$. For such mades the siguificant contribution to the integral is given by $m t^{3} \leq 1$ is that $a>>1 / \gamma$. Eq. (16) takes the form:

$$
\operatorname{Re} \frac{Z(n)}{n}=Z_{0} \int_{0}^{\pi} \alpha d \alpha \sin \left(\frac{n a^{3}}{3}\right)=0.813 \frac{\not}{n^{2 / 3}} .
$$

Here we use the following value of the integral:

$$
\int_{0}^{\pi} d x x^{-1 / 3} \sin x=1.172 \text {. }
$$

Hence, for very large $n$ the impedance rolls off as $n^{-2 / 3}$ For $n<n_{t h}$ inpedance is exponentially small. Tluerefort. the impedance has maximum value at $n=n_{n}$. $V_{n}(y)(1 i)$ and $\mathrm{Eq}$. (5) give:

$$
\left[\mathrm{Re} \frac{Z(n)}{n}\right]_{\max }=223.3 \frac{g}{R} \text { Ohm }
$$

which is reasonable close to the result obtained by axact solution [3]. 
Let us estinate the effect of the finite transverse size $\sigma_{\perp}$ of a bunch. The estimate obtained above is valid if

$$
\sigma_{1}^{2}<<4 R^{2} \sin ^{2}(\alpha) \simeq R^{2} n^{-2 / 3}
$$

For $n \simeq n_{t h}$ that gives $\sigma_{\perp}^{2} \ll R h$. Otherwise, the estimate has to be modified taking into account the finile size $c_{1}$.

\section{CONCLUSION}

The simple approach described the main features of the synchtotion radiation in a vacuum chamber. That opens the possibility of consideration of the effect for more complicated geometries and, bopefully, clarifies the physics of the problem.

\section{ACKNOWLEDGMENTS}

The paper is the result of the discussions during the BD-90 workshop at KEK. Authors are thankful to the orsanjzers of the workshop. One of us (S.H.) eppreciates numerous discussions with $\mathrm{R}$. Warnock and $\mathrm{S}$. Kheifets on the problems considered in the paper.

\section{APPENDIX}

The estimate for the maximun value of the impedance

$$
\left[\operatorname{Re} \frac{Z(n)}{n}\right]_{\max } \simeq 300 \frac{g}{R} \text { Ohn }
$$

can be obtained from the exact solution obtained in the paper by Warnock and Morton [2] (see their Eq. (2.47)) for a particle moving in a cylindrical pillbox cavity:

$$
\begin{aligned}
& \frac{Z(n)}{n}=i \pi^{2} Z_{0} \frac{R}{h} \\
& \times \sum_{\substack{p>1 \\
(\text { od })}}\left[\left(\frac{\alpha_{p}}{\gamma_{p}}\right)^{2} \frac{J_{n}\left(\gamma_{p} R\right)}{J_{n}\left(\gamma_{p} b\right)} p_{n}\left(\gamma_{p} b, \gamma_{p} R\right)\right. \\
&\left.+\left(\frac{i R}{n c}\right) \frac{J_{n}^{\prime}\left(\gamma_{p} R\right)}{J_{n}^{\prime}\left(\gamma_{p} b\right)} s_{n}\left(\gamma_{p} b, \gamma_{p} R\right)\right] .
\end{aligned}
$$

Here $h=2 g$.

$$
\alpha_{p}=\frac{\pi p}{2 g}, \quad \gamma_{p}^{2}=\left(\frac{\omega}{c}\right)^{2}-\alpha_{p}^{2}
$$

and

$$
\begin{aligned}
& p_{n}(x, y)=J_{n}(x) Y_{n}(y)-Y_{n}(x) J_{n}(y), \\
& s_{n}(x, y)=J_{n}^{\prime}(x) Y_{n}^{\prime}(y)-Y_{n}^{\prime \prime}(x) J_{n}^{\prime}(y) .
\end{aligned}
$$

The real part of the impedance is given by the zeros of the denominators. Two terms in Eq. (A1) give equal contribution. Thus, we may consider the first term and double the answer. Expanding denominators near the resonance frequencies

$$
\frac{w}{c}=\sqrt{\left(\frac{\nu_{n}}{b}\right)^{2}+\left(\frac{\pi p}{h}\right)^{2}}
$$

we obtain:

$$
\begin{aligned}
& \operatorname{Re} \frac{Z(n)}{n}=2 \pi^{3} Z_{\bullet} \frac{R}{h} \\
& \quad \times \sum_{p . \nu_{n}}\left(\frac{\pi p c}{\nu_{n} h}\right)^{2} \frac{\nu_{n}}{\omega} \frac{J_{n}^{2}\left(\frac{R}{b} \nu_{n}\right) Y_{n}\left(\nu_{n}\right)}{J_{n}^{\prime}\left(\nu_{n}\right)} \delta\left(\omega-\omega_{\nu, p}\right) .
\end{aligned}
$$

Ilese $\nu_{n}$ are the roots of the Brssel function $J_{n}\left(\nu_{n}\right)=0$. The distance between resonances $\Delta_{\mathrm{L}} / \mathrm{c} \simeq(1 / \mathrm{b}),(\pi / h)$ is much smaller than the threshold frequency $-i t: / r \simeq$ $(1 / h) \sqrt{(R / h)}$. Therefore, the summation may lie rejlartel by the integration. Introducing the polar coorduater (w. क)

$$
\frac{\pi p}{h}=\frac{\omega}{c} \cos \phi, \quad \frac{\nu_{n}}{b}=\frac{\omega}{c} \sin 0
$$

we have

$$
\begin{aligned}
& \text { Re } \frac{Z(n)}{n}=2 \pi^{2} Z_{0} R \frac{\omega}{c} \\
& \times \int_{0}^{\pi} d \phi \frac{\cos ^{2} \phi}{\sin \phi} \frac{J_{n}^{2}(n \sin \phi) Y_{n}\left(n \frac{b}{R} \sin \phi\right)}{J_{n}^{\prime}\left(n \frac{b}{\pi} \sin \phi\right)} .
\end{aligned}
$$

For $n>>1$

$$
J_{n}(n \sin \phi) \simeq \frac{\cos \phi}{\pi \sqrt{3}} K_{1 / 3}\left(\frac{n}{3} \cos ^{3} \theta\right) .
$$

The main contribution is given by small $a \equiv=/ 2-0$

$$
|a| \leq(1 / n)^{1 / 3} \text {. }
$$

For $b>R$ the ratio

$$
\frac{Y_{n}\left(n \frac{b}{h} \sin \phi\right)}{J_{n}^{\prime}\left(n \frac{b}{A} \sin \phi\right)} \simeq 1
$$

Hence.

$$
\operatorname{Re} \frac{Z(n)}{n}=\frac{2}{3} Z_{0} R \frac{\omega}{c} \int_{-\pi / 2}^{\pi / 2} d \alpha \frac{\sin ^{4} \alpha}{\cos \alpha} K_{i / 3}^{2}\left(\frac{n}{3} \sin ^{3} \alpha\right) \text {. }
$$

Replacing $\sin a \simeq a$ and using the integral

$$
\int_{0}^{\infty} d t t^{2 / 3} h_{1 / 3}^{2}(t)=\frac{\sqrt{\pi}}{2^{1 / 3}}
$$

and integrating over $\Delta \omega<<\omega$ we obtain finally:

$$
\text { Re } \frac{Z(n)}{n}=\frac{\sqrt{\pi}}{6^{1 / 3}} \frac{Z_{0}}{n^{2 / 3}} .
$$

Hence, the real part of the impedance rolls off as $n^{-2 / 3}$ for large $n$. The maximun value is reached at the threshold! $n_{t h}$ given by $\mathrm{Eq}$. (5). That gives

$$
\text { Re } \begin{gathered}
\frac{Z(n)}{n}=268 \frac{g}{R} \text { Ohm } . \\
V . \text { REFERENCES }
\end{gathered}
$$

[1] K.Y. $\mathrm{N}_{\mathrm{g}}$ and $\mathrm{R}$. Warnock, "Reactive Impedance of A Smooth Toroidal Chamber Below the Resonance flegions." Phys. Rev. D40, no. 1, p. 231-243. 1989.

[2] R.L. Warnock and P. Morton, "Coherent Synchrotron Riv. diation in a Toroidal Chamber," 5I.AC-PCB-456. Marrh 1988.

[3] R.L. Warnock, "Shielded Coherent Synchrotron Radiatiun and Its Effect on Very Short Bunches," SLAC. P('B- $537 \%$ November 1990.

[4] A.A. Sakolov and I.M. Ternov, "Synchrotron Hicliaticst." Nianka. Moskwa, p. 39, 1966, Eq. (5.2) (in Rusian)! 